\title{
AUTOMATIC SEGMENTATION MYOCARDIAC IMAGES USING MAXIMUM ENTROPY
}

\author{
Mr.Yogesh pawar \\ Prof MIT AOE,
}

Alandi, Pune, India

\author{
Sahil Dhammani \\ MIT AOE,
}

Alandi, Pune,India

\author{
Dnyaneshwar Wable \\ MIT AOE,
}

Alandi, Pune,India

\author{
Mukund Baheti \\ MIT AOE,
}

Alandi,Pune,India

\begin{abstract}
The digital image processing is a widespread applicable technique especially in the area where tools are used for feature extraction and to obtain patterns of studied images. Initially segmentation is used to separate the image into parts that represent an interest object that can be used for further specific study. There are various techniques present which performs such task but a common technique that adapt to all images is required, especially for complex or specific images. Hence our project basically aims to obtain a technique which is convenient for complex and different images. We tend to obtain a more specific result of the input image using histogram quantization, calculating valleys from analysis of histogram slope percentage, calculating threshold using maximum entropy.

This approach provides more specific results over the already proposed technique which will be of great importance to the doctors, pathologists and surgeons to detect the potential cell rejection.
\end{abstract}

Keywords: thresholding, maximum entropy, segmentation, valleys, histogram.

\section{INTRODUCTION}

The goal of segmentation is to simplify and/or change the representation of an image into something that is more meaningful and easier to analyze. The simplest method of image segmentation is called the thresholding method. This method is based on a clip-level (or a threshold value) to turn a gray-scale image into a binary image. In image processing, thresholding is most common method in extracting objects from a picture. If a object is clearly distinguishable from its back ground the gray level histogram will be bimodal and threshold for segmentation can be chosen at bottom of valley. But gray-level histogram is not always bimodal. Methods other than valley seeking are requires to solve this problem.

The key of the method is to select the threshold value (or values when multiple-levels are selected). Several popular methods are used in industry including the Otsu's method (maximum variance), and $\mathrm{k}$-means clustering for detection of threshold.

Histogram based methods are very efficiently when compared to other image segmentation methods because they typically require only one pass through the pixels. In this technique, a histogram is computed from all of the pixels in the image, and the peaks and valleys in the histogram are used to locate the clusters in the image.

The techniques used till dates do not provide a good threshold values that would provide segmentation of entire image. Previous technique proposed a methodology where the algorithm automatically gets the multilevel threshold, by the histogram analysis [1]. The method finds the histogram valleys, which are the places where are concentrated the thresholds and therefore the subdivision of the image. However the method proves effective in cases where the image and the histogram are well defined, for cases where the image is not presented optimally, with noise, distortion and no standardized histograms, the method does not produce an effective threshold that identifies the objects in the image quality. In this context, the paper presents an improved version of the model of multilevel automatic thresholding described in previous methods, considering the group histogram quantization, analysis of the histogram slope percentage and calculation of maximum entropy to define the threshold. These improvements prevent the identification of not significant thresholds and allow more control of the technique during the step of feature extraction in artificial vision systems.

\section{METHODOLOGY}

Histogram-based methods are very efficient when compared to other image segmentation methods because they typically require only one pass through the pixels. In this technique, a histogram is computed from all of the pixels in the image, and the peaks and valleys in the histogram are used to locate the clusters in the image.

The automatic multilevel thresholding method determines the threshold based on the identification of Maximum entropy by comparing the entropies in different regions of the histogram of the image in question. This method is an improvement which allows better control over the thresholds identified [1]. For such, properties were added to the method cited, such as the division of the histogram into classes, analysis of the slope percentages and the determination of the threshold by the calculation of maximum entropy.

The first step in segmentation is to determine the histogram of the image to be studied. The histogram is then divided into classes. A class is one or more gray scale values that make up the histogram. For this division, it is necessary to predefine the class size. If the value informed is 1 , the process analyzes 
each of the intensities of the histogram. For values greater than 1, the increment considered in the iteration process of the method is the predefined value. For instance, consider a class made up of 10 intensities and a histogram with 256 gray levels. The first class encompasses levels 0 to 9 , the second encompasses 10 to 19 , and so forth, totaling 25 classes will 10 levels of gray and one class with six levels (figure 1)

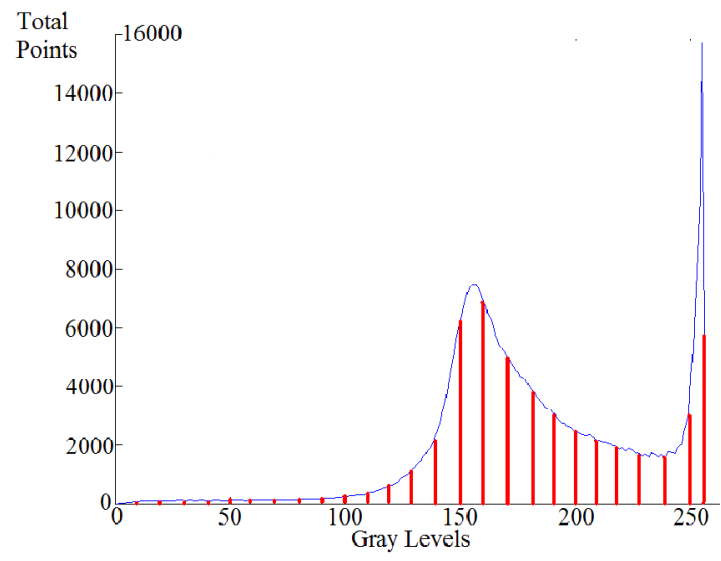

Figure. 1 Example of histogram divided into 26 classes - 25 containing 10 levels and one containing six levels; vertical lines demark classes

Now identify what classes have valleys that are relevant to the specification of the thresholds. The method automatically identifies these classes by means of sign

Transition, analyzing the following characteristics:

- The mean of the points contained in the first half of the class analyzed is compared with the mean of the points in the second half of the same class. If the value of the first half is smaller, the values of the histogram are increasing toward a peak and the sign attributed to the class is positive. Otherwise, a negative sign is attributed to the class, indicating movement toward a valley.

- The sign of the next class is determined. If there is a transition from a negative to a positive sign, the valley is relevant to the specification of a threshold (figure 2).

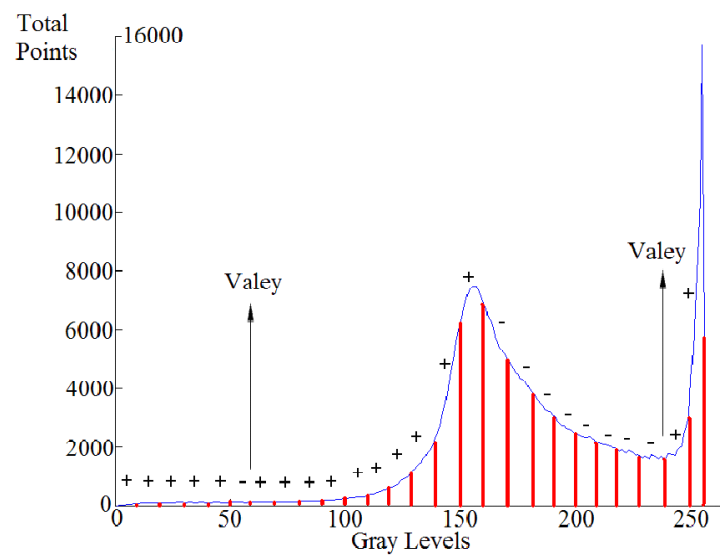

Figure 2. Example of histogram divided into 26 classes with indication of two relevant Valleys to the determination of thresholds one at level 50-59 and another at level 230-239.

The determination of thresholds based on valley analysis may be influenced by homogeneous regions in the histogram (with no valleys or with non-significant valleys). To solve this problem, the proposed method determines that a class is relevant if it has a slope percentage greater than a predefined value. This value can be adjusted to the type of image being studied. A slope percentage is the percentage difference between the means of the points in the first and second halves of the class being analyzed. Two or more classes are grouped together when the slope percentage is lower than the predefined value. This resource allows controlling the sensitivity of the method. A threshold is established when the slope percentage of the class is greater than the predefined value. In the study of images of myocardial biopsies of transplant patients, the values used for the input parameters for segmentation were classes of size 10 and the slope was defined at $35 \%$. These values proved sufficient for the appropriate segmentation of the regions of interest.

Threshold identification using maximum entropy. For each relevant class identified to define a valley, a threshold is calculated based on entropy, considering a probability of an intensity correctly segmenting a given group of objects. For such, the image is taken as the result of a random process in which probability $p$ corresponds to the probability of a pixel in the image taking on an intensity value $i(i=1, . ., n)$ [3], as shown in equations below. The intensity or gray level of the class with the greatest entropy is identified as a threshold.

$$
\begin{gathered}
H=-\sum_{i=1}^{n} p_{i} \log \left(p_{i}\right) \\
p_{i}=\frac{n_{i}}{N}
\end{gathered}
$$

in which $H$ is the entropy of the image; $n$ is the total number of outputs (number of gray levels in the image); $p i$ is the probability of gray level $i$ being found in the image; $n i$ is the number of pixels with intensity $i$; and $N$ is the total number of pixels in the image. The gray level of the group indicated with the highest entropy is identified as a threshold. Completed this phase, the process continues with the analysis of the other groups. To test the technique, we used myocardial images of biopsies from heart transplant. The choice of this type of image to processing is justified by the groups of existing objects, cell core, fibrous tissue, muscle and tissue rejection. 120 images were processed and the results were compared with those provided by the technique presented in Otsu's method.

\section{RESULTS}

In the studied images we used four different input parameters, the image format to be processed, the size of the histogram division group, the filter size, and the percentage of slope to be used for identify thresholds.
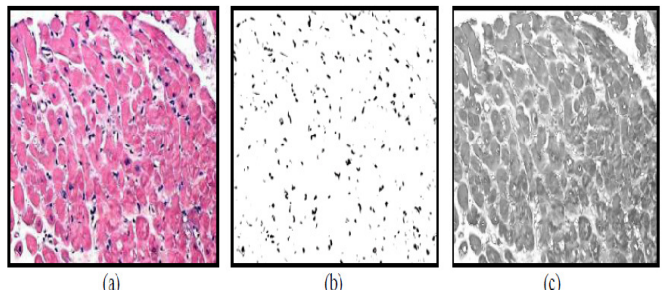
Figure 3: Illustrative case of image obtained from myocardial biopsy of heart-transplant patient

(a) prior to processing; (b) result obtained with proposed segmentation method revealing cell nuclei

and (c) heart muscle (gray) and interstitial spaces (white).

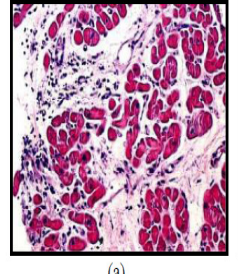

(a)

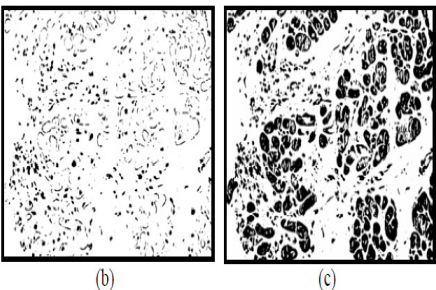

(c)
Figure 4: (a) myocardial biopsy classified as $2 \mathrm{R}$ used to illustrate visual differences obtained with different segmentation methods; (b) cell nuclei segmented using proposed method and (c) using

Otsu's method [2].

\section{REFERENCES}

[1] S. R. Aboud Neta, L. V. Dutra, G. J. Erthal1,

"Limiarização automática em histogramas ultimodais". Proceeedings of the 7th Brazilian Conference on Dynamics, Control and Applications, FCT - Unesp de Presidente Prudente, May, 2008.

[2] Otsu N A 1979 Threshold selection method from graylevel histogram IEEE Transactions on

[3] Pun T 1980 A New Method for Gray-Level Picture Thresholding Using the Entropy of the 